\title{
Socio-economic Factors and Telework Status in the US during the COVID-19 Pandemic
}

\author{
Junfeng Jiao ${ }^{1}$ (D), Amin Azimian ${ }^{1}$ (D) \\ ${ }^{1}$ School of Architecture, University of Texas at Austin \\ Keywords: covid-19, telework, mixed logit, spatial \\ https://doi.org/10.32866/001c.23573
}

Findings

The COVID-19 outbreak posed a considerable risk to the health of people in the US and across the world. To reduce its spread, various companies in America adopted a range of preventive measures, such as telework, for the majority of their workforces. Considering that these measures have disproportionate effects on individuals, this study examined the potential relationship between socioeconomic characteristics and telework status in the United States through mixed logit models developed in this research. Results indicated that telework status was significantly associated with the majority of the variables used in the models, namely, age, gender, educational status, marital status, difficulty with expenses, household size, work type, and anxiety.

\section{Questions}

The spread of COVID-19 posed significant health risks in the US and across the world. To reduce this spread, companies and state authorities adopted various preventive measures early in the pandemic. For instance, many US state governments implemented stay-at-home orders, and many companies allowed employees to work remotely. Regardless of the effectiveness of these preventive measures, they are likely to exert disproportionate effects on individuals with different sociodemographic characteristics. A review of the literature reveals that many studies (Askitas, Tatsiramos, and Verheyden 2020; Bonaccorsi et al. 2020; Jiao and Azimian 2021; Martin et al. 2020; Molloy et al. 2020; Ozili 2020; Saha, Barman, and Chouhan 2020) have assessed the socioeconomic effects of the pandemic over the past year. However, few ones (Kazekami 2020; Shi et al. 2020) have examined the potential relationship between socioeconomic characteristics and telework status during the pandemic. Such information is vital to navigating the current critical situation, as it can inform planners and decision makers about how to effectively support neglected groups.

\section{Methods}

Data were collected through the Household Pulse Survey (US Census, 2020), which was conducted by the US Census Bureau to measure the social and economic effects of the pandemic. The bureau randomly selected a limited number of addresses from across the US to represent the entire population, and these residents received a link to the online survey via email, text, or both. The questionnaire was meant to collect information on respondents' education, demographic characteristics, and transportation use. The survey was conducted in three phases. Phase 1 ran from April 23 to July 21, 2020; phase 2 from August 19 to October 26, 2020; and phase 3 from October 
28 to March 1, 2021. Each phase yielded multiple datasets, each of which contains information collected biweekly from more than 70,000 participants. In this study, we aimed to utilize the latest datasets for each phase, as they capture the latest changes in individuals' travel behaviors for the corresponding phase. However, as the phase 1 questionnaire did not collect information on individuals' telework status, we instead used data from the earliest phase 2 dataset (104,024 participants from August 19-31). Additionally, we used the latest datasets from phase 2 (85,350 participants from October 14-26) and phase 3 (73,858 participants February $17-$ March 1$)$ in the analysis. Table 1 provides the summary statistics of the variables used Models 1, 2, and 3. 


\begin{tabular}{|c|c|c|c|c|}
\hline \multirow{2}{*}{\multicolumn{2}{|c|}{ Variables }} & \multirow{2}{*}{$\begin{array}{l}\text { Model } 1 \\
\text { Frequency \%) }\end{array}$} & \multirow{2}{*}{$\begin{array}{l}\text { Model } 2 \\
\text { Frequency \%) }\end{array}$} & \multirow{2}{*}{$\begin{array}{l}\text { Model } 3 \\
\text { Frequency \%) }\end{array}$} \\
\hline & & & & \\
\hline Dependent variables & $\begin{array}{l}\text { Telework Status } \\
1 \text { If an individual substituted some or all of their typical in-person work for telework because of the coronavirus pandemic. } \\
0 \text { otherwise (base) }\end{array}$ & $\begin{array}{l}45,567(43.2) \\
58,458(56.8)\end{array}$ & $\begin{array}{l}38,231(44.8) \\
47,119(55.2)\end{array}$ & $\begin{array}{l}31,938(43.2) \\
41,920(56.8)\end{array}$ \\
\hline \multirow{10}{*}{ Independent variables } & $\begin{array}{l}\text { Age } \\
\text { Age } 18-35 \text { (base) } \\
\text { Age } 35-50 \\
\text { Age } 50-65 \\
\text { Age } \geq 65\end{array}$ & $\begin{array}{l}17,591(13.4) \\
29,674(25.4) \\
30,057(29.6) \\
26,702(31.6)\end{array}$ & $\begin{array}{l}14,952(17.5) \\
26,416(31.0) \\
24,957(29.2) \\
19,025(22.3)\end{array}$ & $\begin{array}{l}9,913(13.4) \\
18,719(25.4) \\
21,895(29.6) \\
23,331(31.6)\end{array}$ \\
\hline & $\begin{array}{l}\text { Gender } \\
\text { Male ( } 1 \text { if male, } 0 \text { otherwise) } \\
\text { Female (base) }\end{array}$ & $\begin{array}{l}42,869(40.1) \\
61,155(59.9)\end{array}$ & $\begin{array}{l}35,120(41.2) \\
50,230(58.8)\end{array}$ & $\begin{array}{l}29,615(40.1) \\
44,243(59.9)\end{array}$ \\
\hline & $\begin{array}{l}\text { Ethnicity } \\
\text { White ( } 1 \text { if an individual is white, } 0 \text { otherwise) } \\
\text { Non-white (base) }\end{array}$ & $\begin{array}{l}84,584(82.4) \\
19,440(17.6) \\
\end{array}$ & $\begin{array}{l}70,505(82.6) \\
14,845(17.4)\end{array}$ & $\begin{array}{l}60,848(82.4) \\
13,010(17.6)\end{array}$ \\
\hline & $\begin{array}{l}\text { Education } \\
\text { No degree ( } 1 \text { if an individual has no degree, } 0 \text { otherwise) } \\
\text { Associate degree ( } 1 \text { if an individual has an associate degree, } 0 \text { otherwise) } \\
\text { Bachelor's degree ( } 1 \text { if an individual has a bachelor's degree, } 0 \text { otherwise) } \\
\text { Graduate degree (base) }\end{array}$ & $\begin{array}{l}36,681(34.2) \\
10,858(10.6) \\
30,748(29.4) \\
25,737(25.8)\end{array}$ & $\begin{array}{l}28,968(33.9) \\
8,874(10.4) \\
25,529(20.9) \\
21,979(25.8)\end{array}$ & $\begin{array}{l}25,244(34.2) \\
7,850(10.6) \\
21,732(29.4) \\
19,032(25.8)\end{array}$ \\
\hline & $\begin{array}{l}\text { Marital status } \\
\text { Married ( } 1 \text { if married, } 0 \text { otherwise) } \\
\text { Other marital status ( } 1 \text { if divorced/separated/widowed, } 0 \text { otherwise) } \\
\text { Never married (base) }\end{array}$ & $\begin{array}{l}59,826(59.1) \\
22,110(22.8) \\
22,088(18.1)\end{array}$ & $\begin{array}{l}49,562(58.1) \\
18,528(20.2) \\
17,260(21.7)\end{array}$ & $\begin{array}{l}43,620(59.1) \\
16,875(22.8) \\
13,363(18.1)\end{array}$ \\
\hline & $\begin{array}{l}\text { Difficulty with expenses } \\
\text { A little difficult ( } 1 \text { if paying household expenses in the past } 7 \text { days was a little difficult, } 0 \text { otherwise) } \\
\text { Somewhat difficult ( } 1 \text { if paying household expenses in the past } 7 \text { days was somewhat difficult, } 0 \text { otherwise) } \\
\text { Very difficult ( } 1 \text { if paying household expenses in the past } 7 \text { days was very difficult, } 0 \text { otherwise) } \\
\text { Other ( } 1 \text { if unknown or not reported, } 0 \text { otherwise) } \\
\text { Not difficult at all (base) }\end{array}$ & $\begin{array}{l}22,151(21.2) \\
15,271(14.9) \\
10,042(9.6) \\
2,175(3.6) \\
54,385(50.7)\end{array}$ & $\begin{array}{l}17,622(20.7) \\
12,409(14.5) \\
8,542(10.0) \\
1,631(1.9) \\
45,146(52.9)\end{array}$ & $\begin{array}{l}15,652(21.2) \\
11,032(14.9) \\
7,089(9.6) \\
3,660(3.6) \\
37425(50.7)\end{array}$ \\
\hline & $\begin{array}{l}\text { Household size } \\
\mathrm{HH} \leq 2 \text { ( } 1 \text { if household size is } 2 \text { or less, } 0 \text { otherwise) } \\
\mathrm{HH}>2 \text { (base) }\end{array}$ & $\begin{array}{l}54,792(56.3) \\
49,232(43.7)\end{array}$ & $\begin{array}{l}45,691(53.5) \\
39,659(46.5)\end{array}$ & $\begin{array}{l}41,570(56.3) \\
32,288(43.7)\end{array}$ \\
\hline & $\begin{array}{l}\text { Work type } \\
\text { Government ( } 1 \text { if working in government sector, } 0 \text { otherwise) } \\
\text { Private ( } 1 \text { if working in private sector, } 0 \text { otherwise) } \\
\text { Other ( } 1 \text { if unknown or not reported, } 0 \text { otherwise) } \\
\text { Self-employed (base) }\end{array}$ & $\begin{array}{l}11,613(10.2) \\
35,972(31.6) \\
48,641(51.1) \\
7,798(7.1)\end{array}$ & $\begin{array}{l}9,381(11.0) \\
29,664(34.8) \\
39,521(46.3) \\
6,784(7.9)\end{array}$ & $\begin{array}{l}7,552(10.2) \\
23,356(31.6) \\
37,768(51.2) \\
5,182(7.0)\end{array}$ \\
\hline & $\begin{array}{l}\text { Annual household income } \\
<\$ 50,000 \\
\$ 50,000 \text { to } \$ 100,000 \\
>\$ 100,000 \text { (Base) } \\
\text { Other }\end{array}$ & $\begin{array}{l}23,267(22.7) \\
26,567(25.4) \\
31,743(30.2) \\
22,447(21.7)\end{array}$ & $\begin{array}{l}18,946(22.2) \\
21,690(25.4) \\
26,824(31,4) \\
17,890(21.0)\end{array}$ & $\begin{array}{l}16,796(22.7) \\
18,793(25.4) \\
22,277(30.2) \\
15,992(21.7)\end{array}$ \\
\hline & $\begin{array}{l}\text { Anxiousness } \\
\text { Several days } \\
\text { More than half the days } \\
\text { Nearly every day } \\
\text { Other } \\
\text { Not at all (base) }\end{array}$ & $\begin{array}{l}29,885(27.2) \\
11,711(10.0) \\
14,999(14.0) \\
14,977(15.2) \\
32,452(33.6)\end{array}$ & $\begin{array}{l}24,391(28.6) \\
9,589(11.2) \\
13,385(15.7) \\
12,069(14.1) \\
25,916(30.4)\end{array}$ & $\begin{array}{l}20,098(27.2) \\
7,416(10.0) \\
1,0331(14.0) \\
11,218(15.2) \\
24,795(33.6)\end{array}$ \\
\hline
\end{tabular}


Given the categorical nature of our outcome variable (telework status) and the presence of state-level unobserved heterogeneity, we fitted three mixed logit models (with random effect terms) to each of the three datasets. Generally, developing a model with random effect terms is beneficial, as random effect terms can capture the impacts of unmeasured factors (Azimian et al. 2021; Washington, Karlaftis, and Mannering 2010). An example of such a factor in this study is the effect of state policies on travel behaviors.

\section{Findings}

As shown in Table 2, except for ethnicity, which was only significant in Model 1 , all other variables were found to be significant in all three models. Additionally, the direction and magnitude of the relationships are consistent. The results show that the age variable, grouped as 35-50, 50-65, and $\geq 65$, was negatively associated (significant at the .05 level), implying that adults 35 years or older were less likely to work from home than those younger than 35 . Men also exhibited a lower likelihood of working from home than women. Similarly, individuals without graduate degrees were less likely to telework during the pandemic than those with such credentials. As for marital status, while being married was found to be insignificant in the models, reporting an "other marital status (divorced, separated, widowed)" was found to be significantly negatively related, suggesting that such individuals were less likely to work from home than those who had never been married. Individuals who reported some degree of financial difficulty were also less likely to telework compared with those who didn't. In addition, the likelihood of working from home was lower for those in households with one or two people than for those in larger households. 
Table 2. Parameter Estimates in Models 1, 2, and 3 (Base outcome= Not teleworking).

\begin{tabular}{|c|c|c|c|c|c|c|c|c|c|}
\hline \multirow{2}{*}{$\begin{array}{l}\text { Models } \\
\text { Variables }\end{array}$} & \multicolumn{3}{|c|}{ Model 1 (Early Phase 2) } & \multicolumn{3}{|c|}{ Model 2 (Late Phase 2) } & \multicolumn{3}{|c|}{ Model 3 (Late Phase 3) } \\
\hline & Coef. & Odds Ratio & $\mathrm{p}$-value & Coef. & Odds Ratio & $\mathrm{p}$-value & Coef. & Odds Ratio & $p$-value \\
\hline Constant & $1.435^{*}$ & 4.198 & 0.000 & $1.453^{*}$ & 4.276 & 0.000 & $1.571^{*}$ & 3.707 & 0.000 \\
\hline \multicolumn{10}{|l|}{ Age } \\
\hline Age 35-50 & $-0.208^{*}$ & 0.812 & 0.000 & $-0.237^{*}$ & 0.789 & 0.000 & $-0.226^{*}$ & 0.794 & 0.000 \\
\hline Age 50-65 & $-0.359^{*}$ & 0.698 & 0.000 & $-0.491^{*}$ & 0.612 & 0.000 & $-0.355^{*}$ & 0.618 & 0.000 \\
\hline Age $\geq 65$ & $-1.229^{*}$ & 0.293 & 0.000 & $-1.512^{*}$ & 0.220 & 0.000 & $-1.227^{*}$ & 0.223 & 0.000 \\
\hline \multicolumn{10}{|l|}{ Age 18-35 (base) } \\
\hline \multicolumn{10}{|l|}{ Gender } \\
\hline Male & $-0.090^{*}$ & 0.914 & 0.000 & $-0.044^{*}$ & 0.957 & 0.010 & $-0.063^{*}$ & 0.960 & 0.001 \\
\hline \multicolumn{10}{|l|}{ Female (base) } \\
\hline \multicolumn{10}{|l|}{ Ethnicity } \\
\hline White & $-0.049^{*}$ & 0.952 & 0.015 & -0.008 & 0.992 & 0.733 & -0.021 & 0.948 & 0.378 \\
\hline \multicolumn{10}{|l|}{ Non-white (base) } \\
\hline \multicolumn{10}{|l|}{ Education } \\
\hline No degree & $-1.366^{*}$ & 0.255 & 0.000 & $-1.301^{*}$ & 0.272 & 0.000 & $-1.215^{*}$ & 0.274 & 0.000 \\
\hline Associate degree & $-1.143^{*}$ & 0.319 & 0.000 & $-1.136^{*}$ & 0.321 & 0.000 & $-1.013^{*}$ & 0.323 & 0.000 \\
\hline Bachelor's degree & $-0.377^{*}$ & 0.686 & 0.000 & $-0.369^{*}$ & 0.691 & 0.000 & $-0.308^{*}$ & 0.692 & 0.000 \\
\hline \multicolumn{10}{|l|}{ Graduate degree (base) } \\
\hline \multicolumn{10}{|l|}{ Marital status } \\
\hline Married & 0.011 & 1.011 & 0.060 & 0.040 & 1.041 & 0.091 & 0.031 & 1.040 & 0.241 \\
\hline Other marital status & $-0.245^{*}$ & 0.783 & 0.000 & $-0.125^{*}$ & 0.882 & 0.000 & $-0.198^{*}$ & 0.882 & 0.000 \\
\hline \multicolumn{10}{|l|}{ Never married (base) } \\
\hline \multicolumn{10}{|l|}{ Difficulty with expenses } \\
\hline A little difficult & $-0.054^{*}$ & 0.948 & 0.007 & $-0.053^{*}$ & 0.948 & 0.017 & $-0.116^{*}$ & 0.954 & 0.000 \\
\hline Somewhat difficult & $-0.292^{*}$ & 0.747 & 0.000 & $-0.307^{*}$ & 0.736 & 0.000 & $-0.317^{*}$ & 0.743 & 0.000 \\
\hline Very difficult & $-0.691^{*}$ & 0.501 & 0.000 & $-0.660^{*}$ & 0.517 & 0.000 & $-0.622^{*}$ & 0.527 & 0.000 \\
\hline Other & $-0.316^{*}$ & 0.729 & 0.000 & $-0.27^{*}$ & 0.763 & 0.000 & $-0.373^{*}$ & 0.801 & 0.000 \\
\hline \multicolumn{10}{|l|}{ Not at all (base) } \\
\hline \multicolumn{10}{|l|}{ Household size } \\
\hline $\mathrm{HH} \leq 2$ & $-0.360^{*}$ & 0.698 & 0.000 & $-0.272^{*}$ & 0.762 & 0.000 & $-0.411^{*}$ & 0.762 & 0.000 \\
\hline \multicolumn{10}{|l|}{$\mathrm{HH}>2$ (base) } \\
\hline \multicolumn{10}{|l|}{ Work type } \\
\hline Government & $0.843^{*}$ & 2.323 & 0.000 & $0.919^{*}$ & 2.507 & 0.000 & $0.825^{*}$ & 2.517 & 0.000 \\
\hline Private & $0.346^{*}$ & 1.414 & 0.000 & $0.325^{*}$ & 1.384 & 0.000 & $0.253^{*}$ & 1.388 & 0.000 \\
\hline Other & $-0.427^{*}$ & 0.653 & 0.000 & $-0.412^{*}$ & 0.662 & 0.000 & $-0.563^{*}$ & 0.667 & 0.000 \\
\hline \multicolumn{10}{|l|}{ Self-employed (base) } \\
\hline \multicolumn{10}{|c|}{ Annual household income } \\
\hline$<\$ 50,000$ & $-1.374^{*}$ & 0.253 & 0.000 & $-1.402^{*}$ & 0.246 & 0.000 & $-1.298^{*}$ & 0.250 & 0.000 \\
\hline$\$ 50,000$ to $\$ 100,000$ & $-0.659^{*}$ & 0.517 & 0.000 & $-0.684^{*}$ & 0.505 & 0.000 & $-0.624^{*}$ & 0.507 & 0.000 \\
\hline
\end{tabular}




\begin{tabular}{|c|c|c|c|c|c|c|c|c|c|}
\hline Models & \multicolumn{3}{|c|}{ Model 1 (Early Phase 2) } & \multicolumn{3}{|c|}{ Model 2 (Late Phase 2) } & \multicolumn{3}{|c|}{ Model 3 (Late Phase 3) } \\
\hline the & 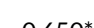 & & & & & & & & \\
\hline \multicolumn{10}{|l|}{ Anxiousness } \\
\hline Several days & $0.423^{*}$ & 1.527 & 0.000 & $0.428^{*}$ & 1.534 & 0.000 & $0.430^{*}$ & 1.545 & 0.000 \\
\hline More than half the days & $0.513^{*}$ & 1.670 & 0.000 & $0.528^{*}$ & 1.696 & 0.000 & $0.518^{*}$ & 1.719 & 0.000 \\
\hline Nearly every day & $0.627^{*}$ & 1.873 & 0.000 & $0.631^{*}$ & 1.879 & 0.000 & $0.570^{*}$ & 1.925 & 0.000 \\
\hline Other & $0.254^{*}$ & 1.289 & 0.000 & $0.259^{*}$ & 1.296 & 0.000 & $0.226^{*}$ & 1.165 & 0.000 \\
\hline \multicolumn{10}{|l|}{ Not at all (base) } \\
\hline $\begin{array}{l}\text { Random-effects parameters } \\
\text { State (Number of groups: } 51 \text { ) } \\
\text { Std.(Constant) }\end{array}$ & 0.262 & - & - & 0.251 & - & - & 0.245 & - & - \\
\hline Log likelihood & \multicolumn{3}{|c|}{-55313.979} & \multicolumn{3}{|c|}{-45328.352} & \multicolumn{3}{|c|}{-39157.755 } \\
\hline Wald Test & \multicolumn{3}{|c|}{$\operatorname{chi} 2(25)=20,740.64 \operatorname{Prob}>\operatorname{chi} 2=0.000$} & \multicolumn{3}{|c|}{$\operatorname{chi} 2(25)=17,376.64$ Prob $>$ chi2 $2=0.000$} & \multicolumn{3}{|c|}{$\operatorname{chi} 2(25)=14,919.75$ Prob $>\operatorname{chi} 2=0.000$} \\
\hline LR test vs. logistic model & \multicolumn{3}{|c|}{ chibar2(01) $=591.63$ Prob $>$ chi2 $=0.000$} & \multicolumn{3}{|c|}{ chibar2(01) $=591.63$ Prob $>$ chi2 $=0.000$} & \multicolumn{3}{|c|}{ chibar $2(01)=423.12$ Prob $>$ chi2 $2=0.000$} \\
\hline
\end{tabular}

Note: * denotes variables significant at $5 \%$. 

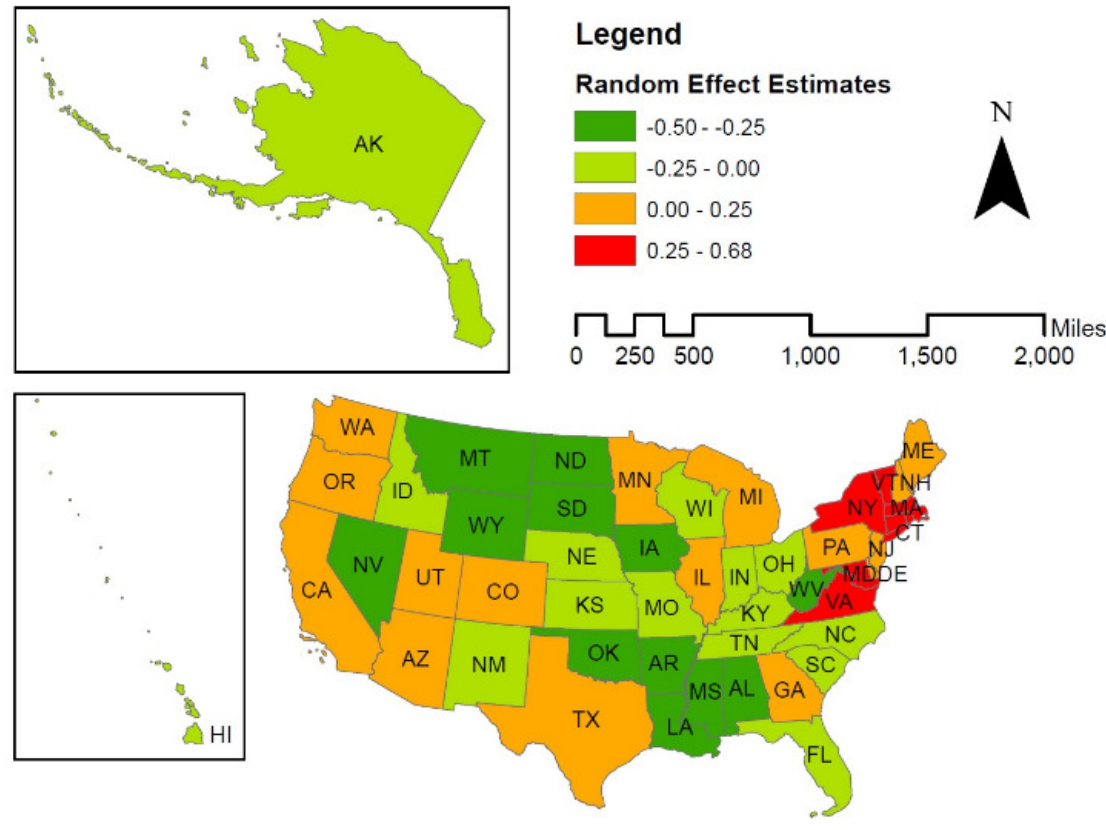

Figure 1. Random Effect Estimates from Model 1 (Early Phase 2)

With regard to work type, those employed by the government or the private sector were more likely than self-employed individuals to engage in telework. Individuals earning an annual income less than $\$ 100,000$ were less likely than those earning more to work from home. Lastly, individuals who experienced anxiety were more inclined to work from home than those who didn't.

The most important component for drawing implications is the random effect term, which captures the variation across states. Figures 1, 2, and 3 show the estimated random effects across the US for early phase 2, late phase 2 , and late phase 3, respectively. The states highlighted in orange and red have positive values, implying that unobserved factors in those states likely incline residents to work from home. The situation is reversed in light and dark green states, which have negative values. In addition, comparing Figures 1 and 2 reveals that between early and late phase 2 , the direction of the random effect values for North Carolina and Ohio changed from negative to positive, while that for Maine changed from positive to negative. Lastly, between late phase 2 and late phase 3 , the random effect terms for four states (Alaska, Maine, Wisconsin, and New Mexico) changed from negative to positive, which could have resulted from state and company policy changes in those states. 


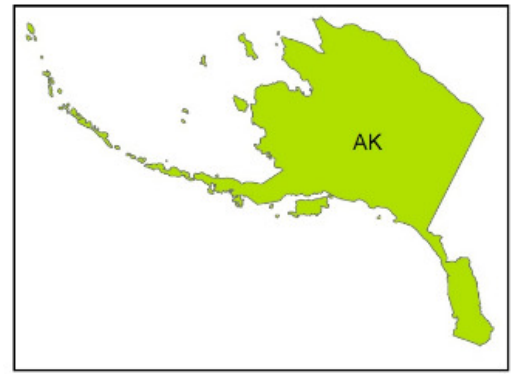

\section{Legend}

\section{Random Effect Estimates}
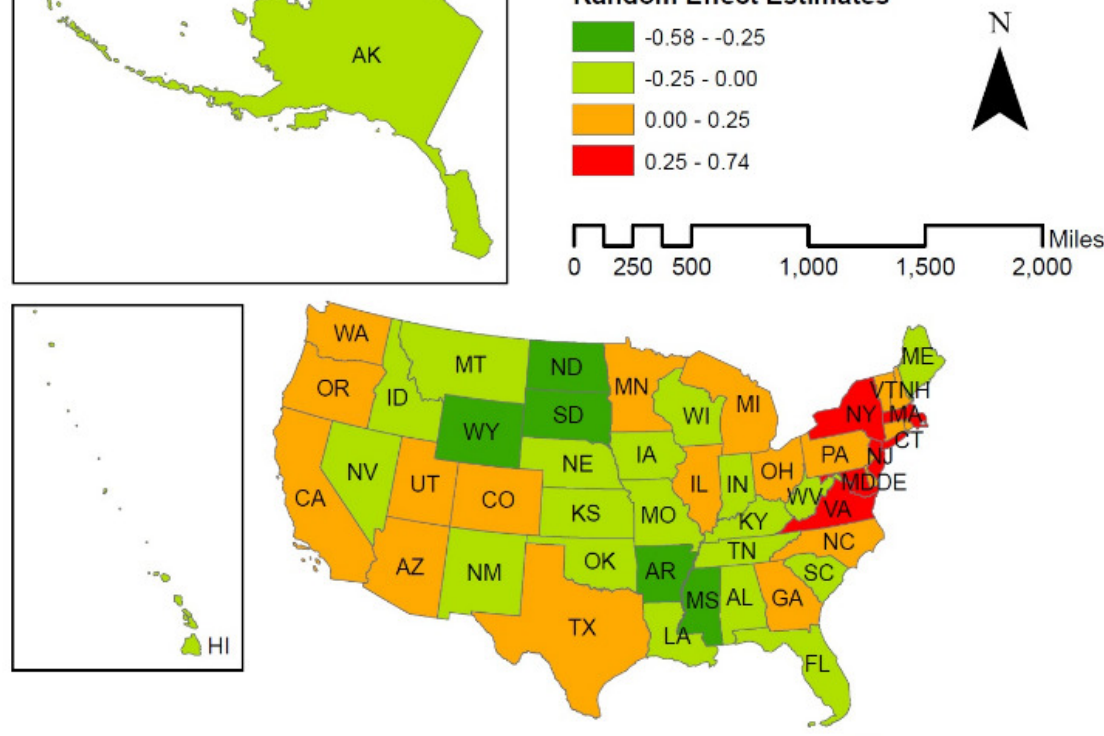

Figure 2. Random Effect Estimates from Model 2 (Late Phase 2)

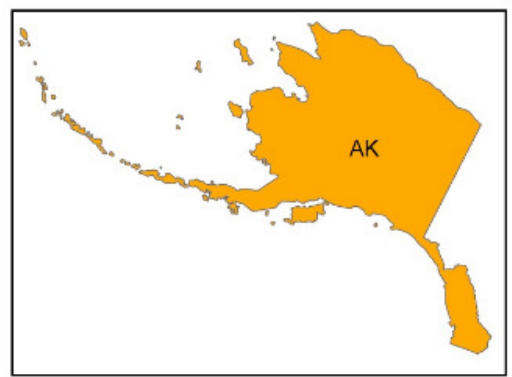

\section{Legend}

Random Effect Estimates
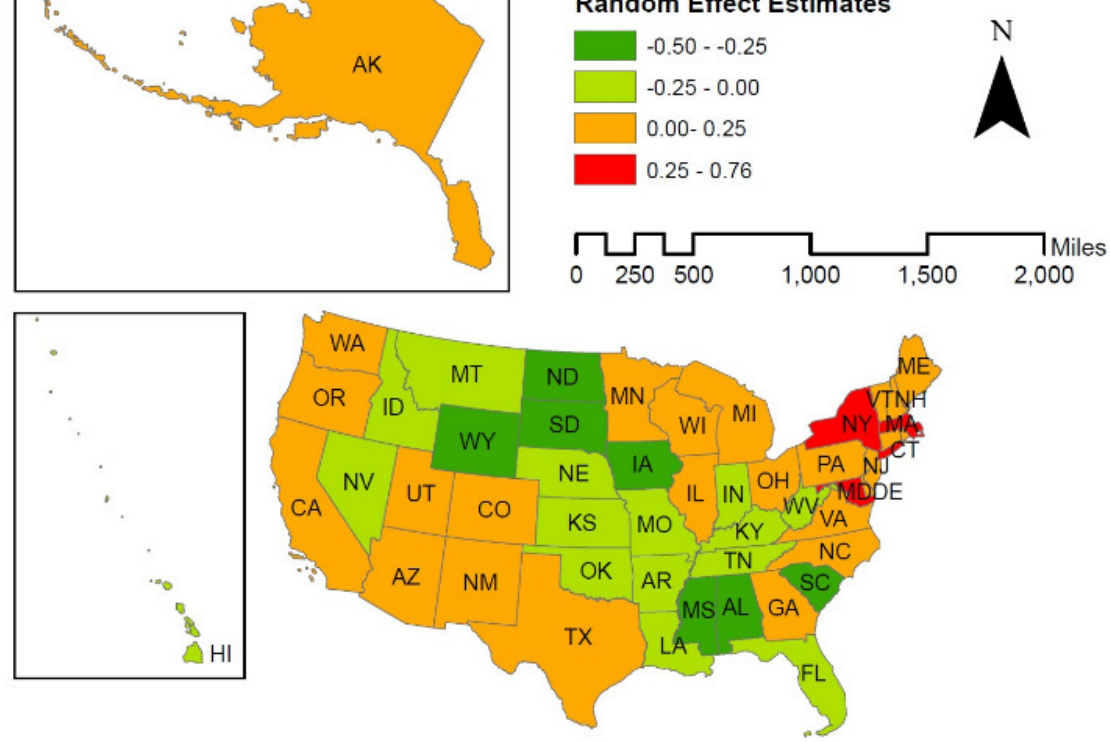

Figure 3. Random Effect Estimates from Model 3 (Late Phase 3)

This is an open-access article distributed under the terms of the Creative Commons Attribution 4.0 International License (CCBY-SA-4.0). View this license's legal deed at https://creativecommons.org/ licenses/by-sa/4.0 and legal code at https://creativecommons.org/licenses/by-sa/4.0/legalcode for more information. 


\section{REFERENCES}

Askitas, Nikos, Konstantinos Tatsiramos, and Bertrand Verheyden. 2020. "Lockdown Strategies, Mobility Patterns and Covid-19.” ArXiv Preprint ArXiv:2006.00531.

Azimian, Amin, V. Dimitra Pyrialakou, Steven Lavrenz, and Sijin Wen. 2021. "Exploring the Effects of Area-Level Factors on Traffic Crash Frequency by Severity Using Multivariate Space-Time Models." Analytic Methods in Accident Research.

Bonaccorsi, Giovanni, Francesco Pierri, Matteo Cinelli, Andrea Flori, Alessandro Galeazzi, Francesco Porcelli, Ana Lucia Schmidt, Carlo Michele Valensise, Antonio Scala, and Walter Quattrociocchi. 2020. "Economic and Social Consequences of Human Mobility Restrictions under COVID-19.” Proceedings of the National Academy of Sciences 117 (27): 15530-35.

Jiao, Junfeng, and Amin Azimian. 2021. "Exploring the Factors Affecting Travel Behaviors During the Second Phase of the COVID-19 Pandemic in the United States." Transportation Letters.

Kazekami, S. 2020. "Mechanisms to Improve Labor Productivity by Performing Telework.” Telecommunications Policy 44 (2): 101868.

Martin, Christopher A, David R Jenkins, Jatinder S Minhas, Laura J Gray, Julian Tang, Caroline Williams, Shirley Sze, Daniel Pan, William Jones, and Raman Verma. 2020. "Socio-Demographic Heterogeneity in the Prevalence of COVID-19 during Lockdown Is Associated with Ethnicity and Household Size: Results from an Observational Cohort Study.” EClinicalMedicine 25: 100466.

Molloy, Joseph, Christopher Tchervenkov, Beat Hintermann, and Kay W Axhausen. 2020. "Tracing the Sars-CoV-2 Impact: The First Month in Switzerland." Transport Findings.

Ozili, Peterson. 2020. "COVID-19 in Africa: Socio-Economic Impact, Policy Response and Opportunities." International Journal of Sociology and Social Policy.

Saha, Jay, Bikash Barman, and Pradip Chouhan. 2020. "Lockdown for COVID-19 and Its Impact on Community Mobility in India: An Analysis of the COVID-19 Community Mobility Reports, 2020." Children and Youth Services Review 116: 105160.

Shi, Xiao, Anne Vernez Moudon, Brian H. Y. Lee, Qing Shen, and Xuegang (Jeff) Ban. 2020.

"Factors Influencing Teleworking Productivity - a Natural Experiment during the COVID-19 Pandemic." Findings, December. https://doi.org/10.32866/001c.18195.

U.S. Census Bureau. 2020. “Household Pulse Survey Public Use File (PUF).” U.S. Census Bureau. U.S. Census Bureau. 2020.

Washington, Simon P, Mattew G Karlaftis, and Fred L Mannering. 2010. "Statistical 427 and Econometric Methods for Transportation Data Analysis.” Taylor E 428. 